\title{
Bit Error Rate Improvement using ESPRIT based Beamforming and RAKE receiver
}

\author{
M. Ali Akbar, Hamza Bin Tila, M. Zulkifl Khalid and Muhammad Asim Ajaz \\ College of Electrical and Mechanical Engineering \\ National University of Sciences and Technology (NUST) \\ Rawalpindi, Pakistan \\ Email: ali.akbar.ceme@gmail.com, hamzabintila@hotmail.com, zulkifl@gmail.com,maaq@ceme.edu.pk
}

\begin{abstract}
The phenomenon of multipath propagation has contributed significantly towards deterioration of quality of signal received in a wireless communication system. Several techniques for multipath mitigation are in use in the current wireless communication technology standards. With the steady rise in the number of wireless devices active in the environment, the concept of beamforming has also gained popularity. In this paper, we propose a smart antenna receiver architecture based on CDMA technology comprising both Rake Receiver (for multipath mitigation) and beamforming on the basis of DOA estimation by ESPRIT algorithm. The purpose is to combine time-space diversity and spatial diversity to achieve better signal reception. The simulation results for the proposed system are presented. The results significantly substantiate our claim of achieving better bit error rate using the proposed receiver.
\end{abstract}

\section{INTRODUCTION}

With the technology advancement in today's society, the ability to communicate with people on the move has evolved remarkably. However, the transmission quality of the signal has deteriorated due to the modernization of the urban cities with skyscrapers and other man-made obstacles. This results in the transmitted signal having to take multiple paths before reaching the intended receiver. Through the multipath transmissions, the signal is severely distorted and attenuated. Methods have to be developed to improve on the signal quality.

\section{A. Motivation}

In most mobile channels, there is more than one propagation path between each transmitter and receiver. Transmitted signals arrive at the receiver via a direct, unobstructed path called line of sight (LOS) path or via multiple paths from the reflection, diffraction and scattering of surrounding objects such as buildings and trees and so a received signal consists of two or more components, each of which traveled a different path from the transmitter. Each multipath component arrives with a delay depending on the path length. Delayed multipath components can cause inter-symbol interference (ISI), and impose an upper limit on the data rate that the channel can support. Fading is another problem in a multipath channel. This "multipath fading" occurs because in general multipath components arrive with different phases. At some points in space, the components cancel each other, causing deep fades in the received signal level. When multiple communications are carried out simultaneously, then in multipath environment, the interference from different directions will also increase. This multipath propagation causes the signal at the receiver to distort and fade significantly, leading to higher bit error rates (BER).

\section{B. Aim \& Objective}

Spread spectrum mobile communication systems use Rake receivers to minimize these communication errors resulting from multipath effects by combining them constructively. To minimize the interference from different directions, smart antennas can be used at the receivers which form the beam in the direction of the incoming multipath and reject the interference coming from other directions.

The main objective of this work is to incorporate the Rake receiver, for multipath mitigation, and ESPRIT based beam forming, for interference rejection, in Direct Sequence Spread Spectrum (DS-SS) Code Division Multiple Access (CDMA) Single-Input-Multiple-Output (SIMO) system so that Bit-ErrorRate (BER) can be reduced in a multipath environment.

The rest of the paper is organized as follows. Section II describes the related work. We present our proposed receiver model in Section III. In Section IV, we describe the simulations carried out to validate the improved performance of our proposed receiver and show the results. Finally, we conclude the paper with an outlook to our future research goals.

\section{RELATED WORK}

The phenomenon of multipath fading in a wireless communication channel has been described in literature ([1],[2],[3],[4]). CDMA technology ([5],[6]) has the potential to solve this problem through adaptive signal processing ([7],[8],[9]) using antenna arrays. In [10] and [11], ESPRIT algorithm has been proposed to achieve spatial diversity in the received signal. The authors in [12] have explored the use of RAKE signals for alignment and recombination of multipaths in a dispersive channel. Our work differs from the existing plethora of research as we attempt to recombine ESPRIT and RAKE receiver in order to achieve spatial as well as temporal diversity.

\section{Proposed Receiver Model}

The receiver has a linear equally spaced antenna array whose output is used by ESPRIT algorithm for estimation of 
Direction of Arrival (DOA) of the desired multipaths. Weights are generated using switched beamforming. After applying weights on the antenna array output, the resulting signal is demodulated from passband to baseband. The baseband signal is used by the Rake receiver for multipath mitigation. After aligning, de-spreading and combining of multipaths by Rake receiver, symbol estimation and channel decoding is done to get the final output signal from the receiver.

\section{A. Adaptive Beamforming}

Beamforming is a signal processing technique used in antenna arrays for directional signal transmission or reception. This spatial selectivity is achieved by using adaptive or fixed receive/transmit beam pattern. The improvement compared with an omni-directional reception/transmission is known as the receive/transmit gain (or loss). Both beam patterns attempt to increase gain in the direction of the user. Adaptive antenna arrays possess an ability to steer beams toward the desired signal, and nulls toward interfering signals, as the user moves around a sector.

In an adaptive array, the phase and amplitude of each element output are controlled by algorithms that iteratively adjust the weight vector of the signals at the array antennas. The weight vectors are controlled depending on the signal and interference as well as the system requirements. These weight vectors are complex in that they provide both amplitude and phase information.

1) Switched Beamforming: The switched beam technique further subdivides sectors into micro-sectors. Each microsector contains a fixed beam pattern, with the greatest gain placed in the centre of the beam. When a mobile user is near a micro-sector, the switched beam system selects the beam with the strongest signal. During a call, the system monitors the signal strength and switches to other fixed beams if necessary. The weights are pre-computed for all the sectors and these are stored in a lookup table. This increases the memory requirements but it significantly improves the speed of beam formation algorithm.

2) DOA Estimation using ESPRIT: ESPRIT (Estimation of Signal Parameters via Rotational Invariance Techniques) is an algorithm for estimation of DOA (Direction of Arrival) of the received signal(s). The idea behind ESPRIT is to divide the array in two equivalent sub-arrays separated by a known displacement $d$. The DOA estimates are then angles of arrival with respect to the direction of $d$. The ESPRIT algorithm is as follows.

1) Obtain a correlation matrix estimate $R_{\tilde{x} \tilde{x}}$ from the received data. Here,

$$
\tilde{x}(t)=\left[\begin{array}{l}
x_{1}(t) \\
x_{2}(t)
\end{array}\right]
$$

is the received signal vector at the antenna array, where $x_{1}(t)$ and $x_{2}(t)$ represent received signals at the first sub-array and at the second sub-array respectively.

2) Compute the eigendecomposition of $R_{\tilde{x} \tilde{x}}$,

$$
R_{\tilde{x} \tilde{x}} E=E \Lambda
$$

where $\Lambda=\operatorname{diag}\left\{\lambda_{1}, \lambda_{2}, \ldots, \lambda_{M}\right\}$ is the matrix containing eigenvalues of $R_{\tilde{x} \tilde{x}}$ and matrix $E=\left\{e_{1}\left|e_{2}\right| \ldots \mid e_{M}\right\}$ consists of eigenvectors of $R_{\tilde{x} \tilde{x}}$. Here $M$ is the number of array elements. We assume that $\lambda_{1} \geq \ldots \geq \lambda_{M}$

3) Obtain the signal subspace estimate by choosing the first $L$ eigenvalues corresponding to the $L$ sources of signals

$$
E_{S}=\left\{e_{1}|\ldots| e_{L}\right\}
$$

and partition $E_{S}$ as,

$$
E_{S}=\left[\begin{array}{ll}
E_{1} & E_{2}
\end{array}\right]^{T}
$$

4) Compute the eigendecomposition of the $2 L \times 2 L$ matrix

$$
\left[\begin{array}{ll}
E_{1}^{H} & E_{2}^{*}
\end{array}\right]^{T}\left[\begin{array}{ll}
E_{1} & E_{2}
\end{array}\right]=V \Lambda V^{H}
$$

5) Partition $V$ into $L \times L$ sub-matrices

$$
V=\left[\begin{array}{ll}
V_{11} & V_{12} \\
V_{21} & V_{22}
\end{array}\right]
$$

6) Calculate the eigenvalues $\psi_{i}$ of the matrix

$$
\Psi=-V_{12} V_{22}^{-1}
$$

for $i=1, \ldots, L$.

7) Estimate the DOA

$$
\widehat{\theta}_{i}=\sin ^{-1}\left(\frac{c}{\omega_{0} d} \arg \left(\psi_{i}\right)\right)
$$

where $d$ is the distance between contiguous antenna elements, $\omega_{0}$ is the carrier frequency and $c$ is the speed of propagation.

\section{B. Rake Receiver}

CDMA systems use the spread spectrum technique with spreading codes designed to provide very low correlation between successive chips. Due to the signal propagation characteristics of the wireless communications channel, the receiver may receive one direct line-of-sight $(\operatorname{LoS})$ wave and many multiple versions of the transmitted signal at a spread of arrival times. If these multipath signals are delayed in time by more than one chip interval, the despreading process will make the uncorrelated noise appear as negligible at the receiver. This leads to the implementation of RAKE receiver within a CDMA system, as it is able to recover each multipath signal and combine them with the correct delays to achieve a significant improvement in the SNR of the output signal. The RAKE receiver however, works only on the basis that these multipath components are practically uncorrelated from one another when their relative propagation delays exceed a chip period.

1) RAKE Receiver Fingers: The basic function of rake receiver can be described as alignment of multipath signals and combination of these aligned multipaths. The rake receiver consists of a stack of fingers each of which aligns and despreads a separate multipath signal. A Rake Receiver having N fingers can align, despread and combine $\mathrm{N}$ different multipath signals. 


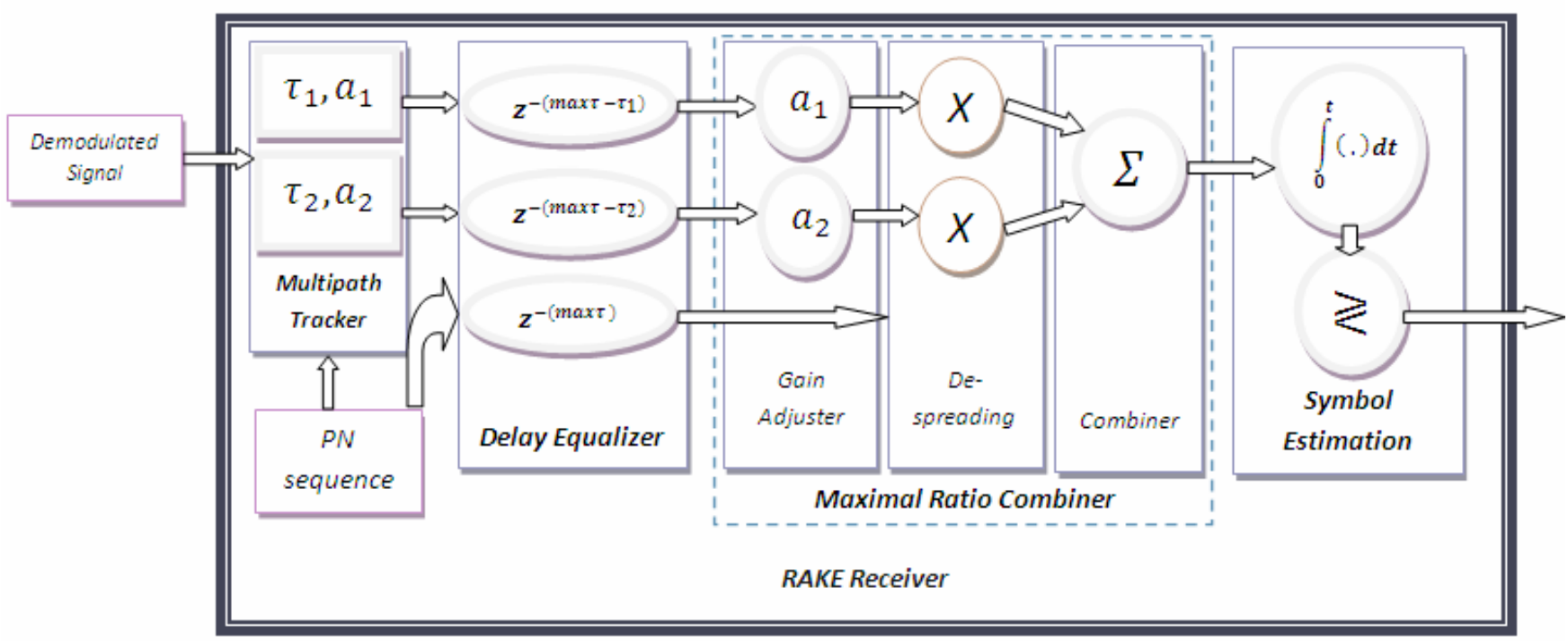

Fig. 1. RAKE Model (with 2 fingers)

2) Basic Model: The basic model of a maximal ratio combiner Rake receiver is given in Figure 1. This model works as follows:

1) The first step is to determine the relative delays and strengths of significant multipath components in the received signal. The multipath tracker block achieves this end by correlating the received signal with the PN sequence generated locally. The peaks in the correlation result represent the multipath signals. The spacing between peaks gives the relative delay and height of peaks gives the strength of that multipath signal.

2) After calculating the parameters (delays and strengths) of the multipath signals, a delay-equalizer block is used to align the selected most significant multipath signals and the PN sequence with the multipath signal with maximum delay.

3) After the alignment of all multipath signals and PN sequence, gain of multipaths is adjusted according to their relative strengths using a gain adjuster block.

4) The multipath signals are then despreaded and combined to achieve a unified signal. This process makes up the maximal ratio combining part.

5) In the end, the unified signal containing the aligned, maximal ratio combined and despreaded multipaths is passed through a low pass filter to calculate mean of a fixed number of samples and then threshold detector is applied. In this way, symbol estimation is performed using probabilistic analysis. The resulting signal is ready to be decoded using channel decoder.

\section{Simulations}

The proposed system simulation model is developed using MATLAB and Simulink. The model is simulated for both single user scenario and multiple user scenario. The simulation model and results are presented below.

\section{A. Single User}

First, we will look into the three basic modules of our communication system, the transmitter, multipath channel and the receiver developed for the single user simulation model. Later on, we describe the process of extending this model to incorporate multiple users. The single user simulation model is described below:

1) DS-SS Transmitter: Direct Sequence Spread Spectrum (DS-SS) Transmitter is the first part of simulation model. In the transmitter, the data is generated, spreaded and modulated.

First of all, random binary data is generated. Then this binary data is converted into Non Return to Zero (NRZ) form where binary bit 1 is represented by -1 and binary bit 0 is represented by 1 . A $64 \times 64$ Walsh function matrix is used to generate the Walsh codes for the simulations. After generation of the Walsh matrix in NRZ form, a random row is selected from the Walsh matrix and assigned to the user as pseudo-noise (PN) sequence. Then the data in NRZ form is spreaded by using the PN sequence so that one data bit is now represented by 64 chips of PN sequence assigned to the user. This is done by multiplying the data bit with the PN sequence. In the end, we modulate the signal using BPSK modulation in which the symbol 1 is represented by the sine wave and symbol -1 is represented by inverted sine wave. In this way the baseband data is converted into the form which is suitable for RF transmission. This data $\left(T_{x}\right)$ is now ready for passband transmission.

2) Channel: After transmission, the signal enters the channel. The channel is dispersive i.e. multipath components of the signal are created. These multipaths may experience different delays depending on the distance they travel while passing through the channel. The amplitude of these multipaths will also vary depending on the route they have taken to reach the receiver. These delays and gains are applied in the simulation model. Additive White Gaussian Noise (AWGN) is added in 
these multipaths in accordance to the SNR of these signals, i.e. for high SNR low noise is added while for low SNR high noise is added. Multipaths may arrive at receiver from different directions. Consequently, they undergo a phase modulation. In the simulation model, we apply this effect by choosing different angles of arrival for different multipaths, and apply the modulation $e^{-j 2 \pi d \sin \theta_{i}}$, where $\theta_{i}$ is the angle with which $i^{t h}$ multipath component arrives at the receiver, and $d$ is the array of relative distances in the receiver array elements.

Mathematically, we can represent the effect of channel as:

$$
R_{x}=\sum_{i=1}^{n}\left(\alpha_{i} \times\left(\left(z^{i} \times T_{x}\right)\right)+n_{i}\right) \times e^{-j 2 \pi d \sin \theta_{i}}
$$

where $T_{x}$ is the transmitted signal; $z^{i}, \alpha_{i}, n_{i}$ and $\theta_{i}$ represent the delay, amplitude gain, AWGN noise and angleof-arrival for the $i^{t h}$ multipath respectively; and $R_{x}$ is the signal received at the receiver antenna array. The total number of multipaths are assumed to be $n$. The channel model is dynamic, i.e. the gains, delays and angles experienced by the multipaths are changing with time.

3) Receiver: This is the final part of the communication system and the most important one. In the receiver the signal is received by forming the beam in the desired signal direction, demodulated and then combined by using RAKE receiver, and probabilistic symbol estimation is performed.

At receiver first of all the direction of arrival of the multipath signals is determined by ESPRIT algorithm. Here the assumption is that the multipath components of the signal are strong enough to distinguish them from noise. Switched beamforming is used to form the beam in the direction of incoming signal. The weights are computed in MATLAB for specific directions by generating the training sequences from different directions and then computing weights for those training sequences by using Least Mean Square (LMS) algorithm. These weights are then stored in the table. When the DOA of the signal is known, the weights in that specific direction are looked up from the table and by multiplying those weights with the incoming signal the beam is formed in that direction maximizing the gain in that direction and minimizing the interferences from other directions. The beam formed for a signal containing two multipaths from user 1 with angles of arrival $30^{\circ}$ and $45^{\circ}$, and an interference signal from user2 with angle of arrival $-60^{\circ}$ is shown in Figure 2.

After reception of the multipath signal components, they are demodulated. For demodulating the signal it is again multiplied with the same sine wave as was used during the modulation process. Then a low pass filter is applied. The demodulated multipath signals are passed through the rake receiver. The rake receiver computes the delays of different multipath components and combines them constructively. The delays of the different multipath components are found by performing the correlation of WALSH code with the received signal. Same WALSH code is used in the receiver as was used in the transmitter. After finding the delays the multipath signals are aligned with the multipath with largest delay. The aligned

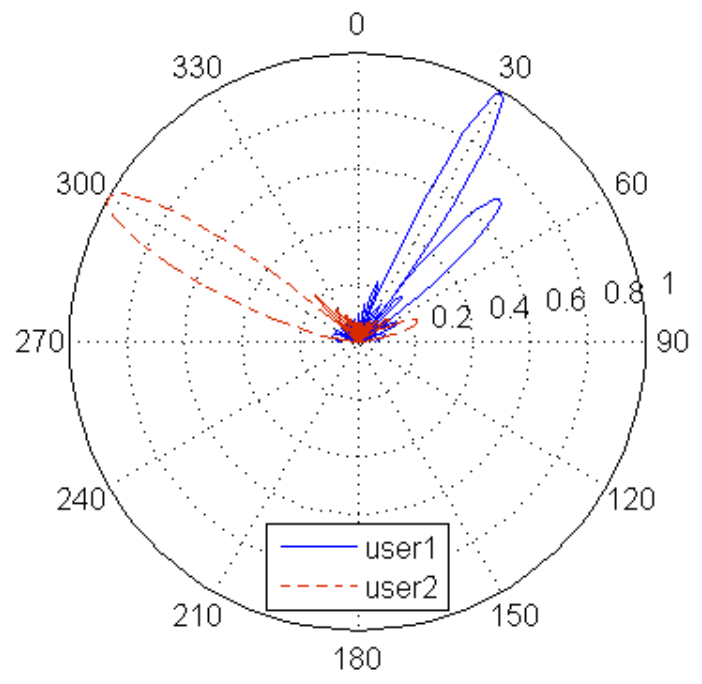

Fig. 2. Switched Beam-formation

multipaths are then despreaded by multiplying them with the WALSH code. These signals are given the specific gains by multiplying them with the corresponding peaks found in the correlation process. These gains enhance the strong multipath signals and suppress the weak multipath components. After adjusting the gains these signals are combined and we get the output of the rake receiver in which the multipath signals are combined in order to have higher SNR. In our system we have rake receiver which combines two strongest multipath signals of a user.

In the end the symbol estimation is performed by using the probabilistic analysis in which the average of the signal over 64 samples is taken and on the basis of that mean value the decision is taken whether the symbol is 1 or -1 . In the end the signal is converted back to binary form to get the original data.

4) Results: The results for Bit Error Rate (BER) for different Signal to Noise Ratio (SNR) values for the proposed system (single user) are shown in Figure 3. The results show a significant improvement in performance of receiver when the proposed additions are made in the traditional DS-SS receiver.

\section{B. Multiple Users}

Now to incorporate multiple users in this system, we increase the number of correlators in our system. The signals of multiple users can be distinguished from the unique PN sequence for each user. For two users we have two correlators at the receiver and we can separate two multipath signals of each user. Similarly for three users we have three correlators which enable us to separate two multipath signals for each of the three users.

1) Results: The results for Bit Error Rate (BER) for different Signal to Noise Ratio (SNR) values for the proposed system (for multiple users) are shown in Figure 4. The results show that the proposed system is able to maintain its improved performance when multiple users access the system 


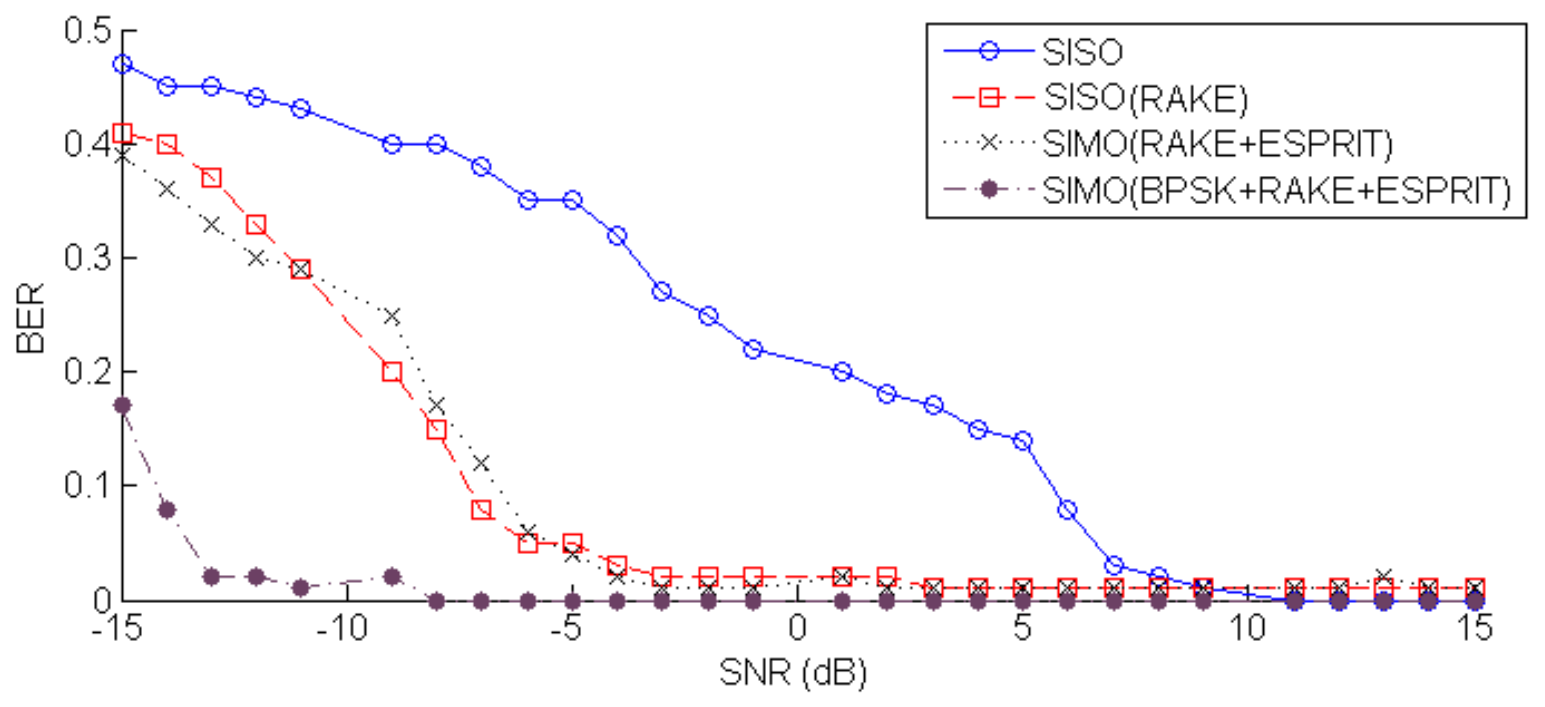

Fig. 3. BER result for single user scenario

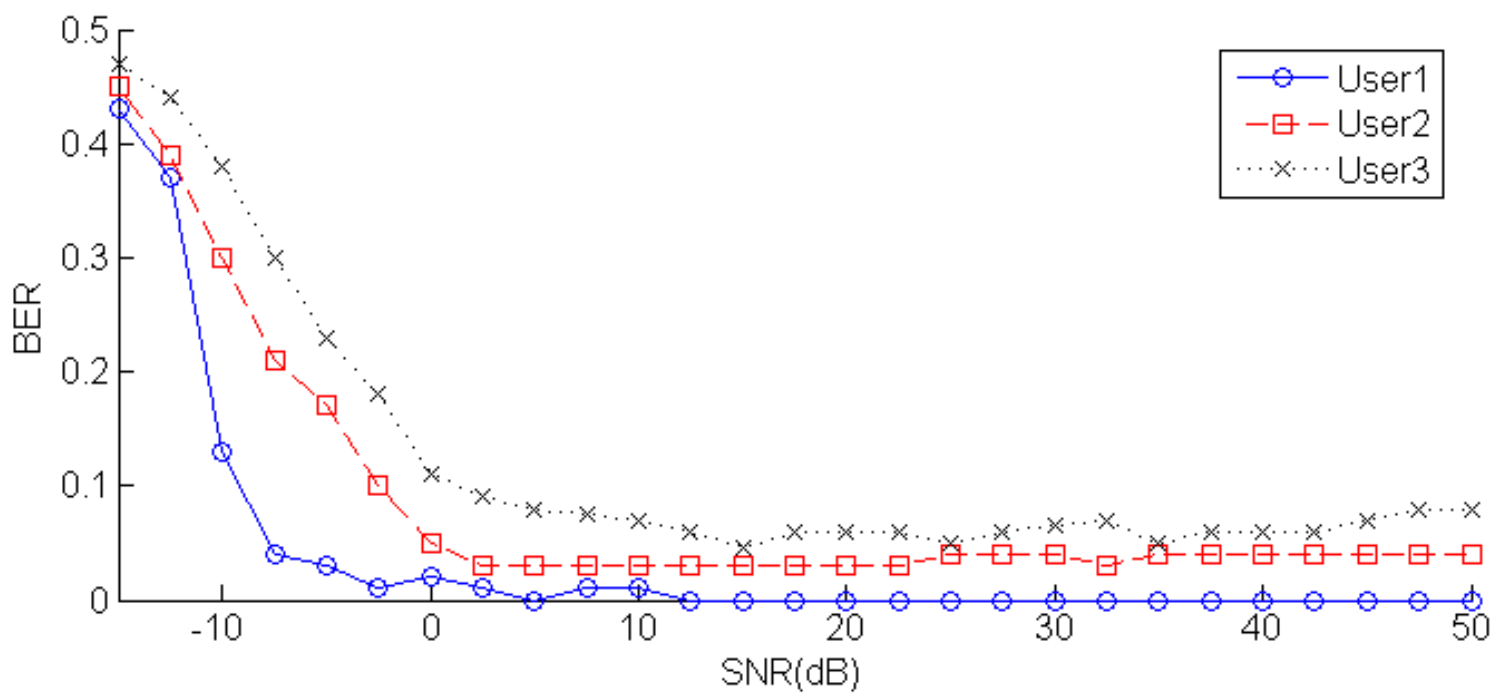

Fig. 4. BER result for multiple user scenario (proposed receiver - SIMO (BPSK+RAKE+ESPRIT) — is used)

via CDMA technique. The fluctuations in the results at high SNRs are due to the variations in channel whose characteristics are dynamic with respect to time.

\section{Graphical User Interface}

The graphical user interface for this system is also developed which at present is for single and two users. It helps in simulating and testing the proposed system for different channel scenarios.

The parameters for the channel that can be controlled via the graphical user interface are:

1) Direction of Arrival (DOA) for the multipath signals

2) Path Delays for different Multipaths

The GUI provides options to the user to run simulation for both single user scenario and multiple user scenario. Currently, multiple user scenario simulation via GUI is limited to two users; however it can be increased through minor additions in the interface and Simulink Model.

After specifying the required simulation parameters, the user can simulate the scenario for our proposed system. The simulation results are displayed when the simulation is completed. The results displayed to the user via GUI are:

1) Plot of Transmitted Signal on Time-Amplitude axis for each user

2) Plot of Received Signal on Time-Amplitude axis for each user

3) Polar plot of beam formed on receiver antennas in the direction of multipaths

4) Bit Error Rate (BER) results for each user

A snap shot of GUI is shown in Figure 5. 


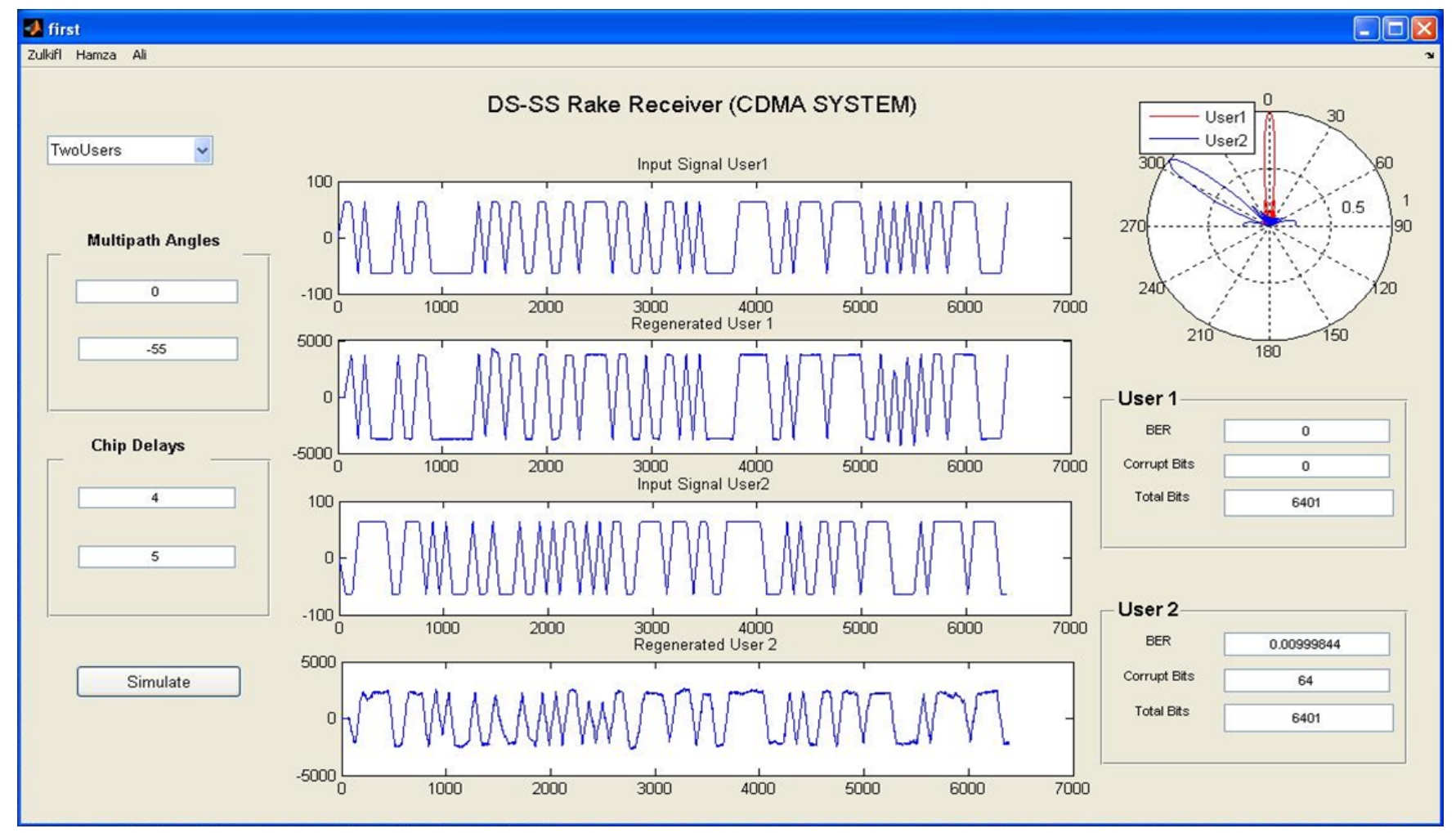

Fig. 5. Graphical User Interface

\section{CONCLUSION \& Future WORK}

In this paper, we proposed smart antenna receiver architecture based on CDMA technology comprising both Rake Receiver (for multipath mitigation) and beamforming on the basis of DOA estimation by ESPRIT algorithm. The purpose was to combine time-space diversity and spatial diversity to achieve better signal reception. We successfully simulated the traditional DS-SS system and the proposed system using Matlab and Simulink. Scenarios for single user as well as multiple users were simulated. Graphical User Interface was also developed to automate the task of simulation and present the results in a systematic manner. We obtained simulation results for BER vs SNR in single user DS-SS and multi-user CDMA system. The results showed that our proposed system had a remarkably improved signal reception as compared to traditional systems.

Future work should focus on physical implementation and extensive testing of this system with RF front-end in realworld environment. A test-bed with RF front-ends can be developed for rigorous testing of the proposed architecture. The system is currently Single-Input-Multiple-Output (SIMO). Using OFDM, it can be converted into Multiple Input Multiple Output (MIMO) architecture with remarkable increase in channel capacity. Furthermore, OFDMA can be used to get a MIMO-MU (multiuser) system. Channel equalization, carrier recovery and phase recovery techniques should also be incorporated in the system to avoid possible phase reversal of multipath signals in the Rake Receiver fingers.

\section{REFERENCES}

[1] T. Rappaport, Wireless communications: principles and practice. Prentice Hall, NJ, USA, 2002.

[2] W. Stallings, Wireless communications and networks. Prentice Hall, NJ, USA, 2004

[3] L. Couch, Digital and analog communication systems. Prentice Hall, NJ, USA, 2001.

[4] R. Blake, Comprehensive electronic communication. West Publishing Company, Minneapolis, 1997.

[5] R. Prasad, CDMA for wireless personal communications. Artech House, Inc. MA, USA, 1996.

[6] C. D. Group, "CDMA Technology: The Drive towards Third Generation (3G) Systems," 2002, http://www.cdg.org/.

[7] B. Widrow and S. Stearns, Adaptive signal processing. Prentice Hall, NJ, USA, 1985.

[8] S. Haykin, Adaptive filter theory. Prentice hall, NJ, USA, 2002.

[9] Y. Wang and J. Cruz, "Adaptive antenna arrays for cellular CDMA communication systems," in Proc. Internat. Conf Acoustics, Speech, and Signal Processing, vol. 3. IEE, 1995, pp. 1725-1725.

[10] R. Roy and T. Kailath, "ESPRIT-estimation of signal parameters via rotational invariance techniques," IEEE Trans. on Acoustics Speech and Signal Processing, vol. 37, no. 7, pp. 984-995, 1989.

[11] R. Roy, A. Paulraj, and T. Kailath, "ESPRIT-A subspace rotation approach to estimation of parameters of cisoids in noise," IEEE Trans. on Acoustics, Speech and signal processing, vol. 34, no. 5, pp. 13401342, 1986.

[12] C. Brunner, M. Haardt, and J. Nossek, "On space-time rake receiver structures for WCDMA," in Signals, Systems, and Computers, 1999. Conference Record of the Thirty-Third Asilomar Conference on, vol. 2, 1999. 